\title{
Ultra-Strong Machine Learning: comprehensibility of programs learned with ILP
}

\author{
Stephen H. Muggleton' ${ }^{1}$ (D) - Ute Schmid ${ }^{2}$ - Christina Zeller ${ }^{2}$ • \\ Alireza Tamaddoni-Nezhad ${ }^{3}$. Tarek Besold $^{4}$
}

Received: 31 March 2017 / Accepted: 5 April 2018 / Published online: 7 May 2018

(C) The Author(s) 2018

\begin{abstract}
During the 1980s Michie defined Machine Learning in terms of two orthogonal axes of performance: predictive accuracy and comprehensibility of generated hypotheses. Since predictive accuracy was readily measurable and comprehensibility not so, later definitions in the 1990s, such as Mitchell's, tended to use a one-dimensional approach to Machine Learning based solely on predictive accuracy, ultimately favouring statistical over symbolic Machine Learning approaches. In this paper we provide a definition of comprehensibility of hypotheses which can be estimated using human participant trials. We present two sets of experiments testing human comprehensibility of logic programs. In the first experiment we test human comprehensibility with and without predicate invention. Results indicate comprehensibility is affected not only by the complexity of the presented program but also by the existence of anonymous predicate symbols. In the second experiment we directly test whether any state-of-the-art ILP systems are ultra-strong learners in Michie's sense, and select the Metagol system for use in humans trials. Results show participants were not able to learn
\end{abstract}

Editors: James Cussens and Alessandra Russo.

Stephen H. Muggleton

s.muggleton@imperial.ac.uk

Ute Schmid

ute.schmid@uni-bamberg.de

Christina Zeller

christina.zeller@uni-bamberg.de

Alireza Tamaddoni-Nezhad

a.tamaddoni-nezhad@imperial.ac.uk ; a.tamaddoni-nezhad@surrey.ac.uk

Tarek Besold

tbesold@uni-bremen.de

1 Department of Computing, Imperial College London, London, UK

2 Cognitive Systems Group, University of Bamberg, Bamberg, Germany

3 Department of Computer Science, University of Surrey, Guildford, UK

4 Digital Media Lab, University of Bremen, Bremen, Germany 
the relational concept on their own from a set of examples but they were able to apply the relational definition provided by the ILP system correctly. This implies the existence of a class of relational concepts which are hard to acquire for humans, though easy to understand given an abstract explanation. We believe improved understanding of this class could have potential relevance to contexts involving human learning, teaching and verbal interaction.

Keywords Inductive logic programming · Comprehensibility · Ultra-strong machine learning

\section{Introduction}

In a recent paper (Schmid et al. 2017) the authors introduced an operational definition for comprehensibility of logic programs and conducted human trials to determine how properties of a program affect its ease of comprehension. This paper builds on and extends the earlier work by investigating whether machines can not only learn new concepts but explain those concepts to humans thereby improving human task performance. The definition of comprehensibility allows, for the first time, experimental demonstration of Donald Michie's Ultra-Strong Machine Learning criterion.

Michie (1988) provided weak, strong and ultra-strong criteria for Machine Learning. Michie's aim was to provide operational criteria for various qualities of machine learning which include not only predictive performance but also comprehensibility of learned knowledge. His weak criterion identifies the case in which the machine learner produces improved predictive performance with increasing amounts of data. The strong criterion additionally requires the learning system to provide its hypotheses in symbolic form. Lastly, the ultrastrong criterion extends the strong criterion by requiring the learner to teach the hypothesis to a human, whose performance is consequently increased to a level beyond that of the human studying the training data alone.

Most of modern Machine Learning can be viewed as consistent with Michie's weak criterion. By contrast, the strong criterion plays an ongoing role within the context of Inductive Logic Programming. However, to date no documented attempt has been made, even within Inductive Logic Programming, to apply, or demonstrate Michie's ultra-strong criterion to a Machine Learning system. As argued in Schmid et al. (2017) the major barrier has been the lack of an operational notion of human comprehension of symbolic concepts.

Within Artificial Intelligence (AI) comprehensibility of symbolic knowledge is viewed as one of the defining factors which distinguishes logic-based representations from those employed in statistical Machine Learning. In Schmid et al. (2017) the issue is addressed by introducing a definition of comprehensibility which is inspired by "Comprehension Tests", administered to school children. Such a test (see Fig. 1) comprises the presentation of a piece of text, followed by questions which probe the child's understanding. Answers to questions in some cases may not be directly stated, but instead inferred from the text. Once the test is scored, the proportion of questions correctly answered provides the measure of a pupil's textual comprehension.

In the same fashion, our operational definition of comprehensibility is based on presentation of a logic program to an experimental participant (see Fig. 2), who is given time to study it, after which the score is used to assess their degree of comprehension. Understanding the declarative reading of a logic program is directly analogous to understanding a piece of natural language text. Such understanding can be tested in a similar fashion by questioning a human reader about the factual content and implied consequences of the statements in 
For many years people believed the cleverest animals after man were chimpanzees. Now, however, there is proof that dolphins may be even cleverer than these big apes.

Question: Which animals do people think may be the cleverest?

Fig. 1 Text comprehension test (Credit: http://englishteststore.net)

Fig. 2 Program comprehension

test

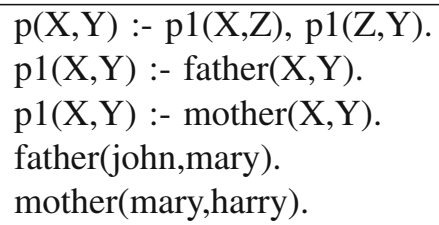

Question: p(john,harry)?

the logic program. The existence of a declarative reading of pure logic programs provides a justification for investigating the relative comprehensibility of machine learned hypotheses within Inductive Logic Programming. The detailed results of such a logic program test can be used to identify factors in a logic program which affect its comprehensibility both for individuals and for groups of participants. The existence of an experimental methodology for testing comprehensibility has the potential to provide empirical input for improvement of Machine Learning systems for which the generated hypotheses are intended to provide insights.

The paper is arranged as follows. In Sect. 2 we discuss existing work relevant to the paper. The framework, including relevant definitions and their relationship to experimental hypotheses is described in Sect. 3. Section 4 describes two experiments involving human participants. The first experiment tests the degree to which predicate invention affects human comprehensibility of concepts. The second experiment tests whether an ILP system can pass Michie's Ultra-Strong Learning criterion. Finally in Sect. 5 we conclude the paper and discuss further work.

\section{Related work}

This section offers framing information concerning research into comprehensibility and explainability of systems in general, and familiarizes the reader with the core notions motivating our work in particular. We first present a short overview of related lines of investigation in AI and Machine Learning, respectively, before specifically discussing cognitive and computational aspects of predicate invention in the context of the hierarchical structuring of complex concepts, and of induction, abduction, and recursion as important mechanisms for concept formation and representation.

\subsection{Comprehensibility and explanation in AI}

Studies of the comprehensibility, and relatedly explainability, of computational systems have a long tradition, dating back at least to research into expert and decision support systems in 
the early 1980s. Clancey (1983) questioned whether expert knowledge can be encoded as a uniform, weakly-structured set of if/then associations [as, e.g., done in the MYCIN system (Shortliffe 1974)] when rules are modified by people other than the original author, or used in teaching (i.e., support active learning). Consequently, efforts were undertaken to improve expert system explanations (see, e.g., Chandrasekaran et al. 1989; Wick and Thompson 1992). One resulting line of work addressed the representation formalisms used (Gaines 1996). Among others things, statements were taken to be more comprehensible when they were either textually smaller or more coherent. Moreover, compositions of familiar concepts were taken as easier to understand.

In Van Someren (1995), it was suggested that computational systems should be thought of as consisting of two inter-related components: one for problem-solving and another for communication. It was further suggested these components require separate inter-related representations. Askira-Gelman's (1998) meta-study reaches a similar conclusion when suggesting that a separation between low-level mechanisms and external representation, together with an addition of conversion capabilities, allow increased flexibility. Additionally, the importance of supporting the user on a semantic level (e.g., through consistency of results with pre-existing domain knowledge) was reaffirmed.

In the context of AI testing and evaluation the importance of human comprehensibility of intelligent systems has recently been emphasised by Forbus (2016). For his software social organisms, comprehensibility of the system's behaviour and outputs is paramount, since only efficient communication enables participation in human society. In general when looking at the original Turing Test (Turing 1950) and discussions of new and updated versions or substitutes for it, comprehensibility plays a crucial role. While there are frequently suggestions to abandon the Turing Test and focus on more clearly specified tasks in well-defined domains, putting emphasis on making systems and their output comprehensible for humans offers an alternative approach to overcoming limitations of the original test, while still maintaining domain and task generality.

\subsection{Comprehensibility and explanation in Machine Learning}

In Machine Learning, comprehensibility has been discussed in the context of ArgumentBased Machine Learning (ABML) (Mozina et al. 2007), which applies methods from argumentation in combination with a rule-learning approach. Explanations provided by domain experts concerning positive or negative arguments are included in the learning data and serve to enrich selected examples. Although ABML enhances the degree of explanation, it still fails to pass Michie's ultra-strong test since no demonstration of user comprehensibility of learned hypotheses is guaranteed. Moreover, questions and discussions about comprehensibility have also entered the study of classification models (Freitas 2014; Letham et al. 2015; Lipton 2016). However, while the need for comprehensibility is emphasized, no definitive test of the kind provided by our definition in Sect. 3 is offered.

Another approach which engages with aspects of comprehensibility, logical reasoning and to some extent, predicate invention (i.e., the automated introduction of auxiliary predicates), discussed in more detail in the next subsection due to its role in our first experiment in Sect. 4, is Explanation-Based Learning (EBL) (e.g. Mitchell et al. 1986). EBL uses background knowledge in a mainly deductive inference mechanism to "explain" how each training example is an instance of the target concept. The deductive proof of an example yields a specialisation of the given domain theory leading to the generation of a special-purpose sub-theory described in a user-defined operational language. The learning process in EBL is comparable to the use of proof-completion in the context of Meta-Interpretive Learning (MIL) (Muggleton et al. 
2014, 2015), with EBL assuming a complete (first-order) domain theory and using deduction (rather than induction or abduction) as key differences. Some EBL systems can discover new features not explicit in the training examples but required in the general rule describing the former. For example, Prolog-EBG (Kedar-Cabelli and McCarty 1987) automatically formulates meaningful constraints required in the rules underlying the training examples. EBL is also realised in the inductive functional programming system Igor where observed program traces are explained as unfolding of an unknown recursive function following some program scheme (Kitzelmann and Schmid 2006).

However, existing EBL systems do not pass Michie's ultra-strong test either: again there is no guarantee of user comprehensibility of learned hypotheses. The deductively generated syntactic explanations (i.e. formal proofs) could be far from human comprehensible explanations in a semantic sense (causal, mechanistic, etc.).

Interpretable systems currently also constitute a topic of increased interest in the study of artificial neural networks. In this context, a system is considered interpretable if "a user cannot only see, but also study and understand how inputs are mathematically mapped to outputs" (Doran et al. 2017), with regression models (Schielzeth 2010) or generalized additive models (Lou et al. 2012) serving as examples. However, as discussed for instance in Vellido et al. (2012), Rudin (2014), interpretability in these cases refers almost exclusively to a mathematical property of the models, allowing for a certain degree of knowledge extraction from the model and subsequent interpretation by domain experts. This departs from our targeted notion of comprehensibility as a direct property of a system or representation not requiring an active step of interpretation or technical understanding of the aforementioned mapping of inputs to outputs.

\subsection{Empirical evaluations of comprehensibility of models and explanatory value of outputs}

Questions and discussions about comprehensibility have also entered the study of classification models (Freitas 2014; Letham et al. 2015; Lipton 2016). However, while the need for comprehensibility is emphasized, few studies attempt empirical evaluations, and more generally no definitive test of the kind provided by our definition in Sect. 3, in conjunction with the subsequent evaluation methodology, is offered. Allahyari and Lavesson (2011) present a study targeting the understandability of classification models in the context of data mining from a user-centered perspective. As part of a survey-based assessment, participants are asked to rank different models according to their understandability. Among others, the results indicate that decision tree models are perceived as more understandable than rulebased models. Also, in one of two study cases a negative correlation between the complexity and the understandability of the classification models is found. In a more extensive empirical study, again in the context of data mining, Huysmans et al. (2011) investigate the suitability of a number of alternative representation formats for classification when interpretability is a key requirement. In order to compare different standard representation formats, an end-user experiment is designed to test the accuracy, response time, and answer confidence for a set of problem-solving tasks. Analysis of the results shows that in the tested setting decision tables perform significantly better on all criteria, and post-test voting additionally indicates a clear preference of users for decision tables in terms of ease of use. In Lakkaraju et al. (2016), in the context of introducing decision sets as a more interpretable way of organizing classification rules, a user study is also reported which assessed the difficulty of interpreting the introduced models. The study compares decision sets and decision lists using two types of tasks: answering multiple-choice questions about the decision boundaries of the models and 
writing descriptions of classes based on them. The results show that the structure of decision sets enables users to better understand the predictions they will make, indicated by more accurate and faster answers to multiple-choice questions about the decision boundaries of a decision set versus a decision list. Also, humans are found to be three times more accurate when asked to write class descriptions given a decision set versus a decision list, while using significantly shorter descriptions and requiring less time.

In a context more reminiscent of the already mentioned work on the explainability of expert systems, Lim et al. (2009) address the intelligibility of context-aware intelligent systems when automatically providing explanations about a system's decision process. In order to evaluate the effectiveness of different types of explanations, participants are shown examples of a system's operation along with various automatically generated explanations, and then are tested on their understanding of the system. The results show, among others, that explanations describing why the system behaved a certain way result in better understanding and stronger feelings of trust on the user side. Explanations describing why the system did not behave a certain way, result in lower understanding yet adequate performance.

\subsection{Hierarchical structuring of complex concepts through predicate invention}

Research into the inner structure of complex concepts found these to be strongly hierarchically organised in a tree-like structure, with more general categories higher in the hierarchy dominating lower-level categories via IS-A links (Murphy and Lassaline 1997). This hierarchical structure is presumably acquired by successive abstractions based on sets of instances from lower-level categories. Emulating these generalisation processes, predicate invention has been viewed as an important problem since the early days of ILP (e.g. Muggleton and Buntine 1988; Rouveirol and Puget 1989; Stahl 1992), though limited progress has been made in this topic recently (Muggleton et al. 2011). Early approaches were based on the use of $W$-operators within the inverting resolution framework (e.g., Muggleton and Buntine 1988; Rouveirol and Puget 1989). However, the completeness of these approaches was never demonstrated, partly because of the lack of a declarative bias to delimit the hypothesis space. Failure to address these issues has, until recently, led to limited progress being made in this important topic and many well-known ILP systems such as ALEPH (Srinivasan 2001) and FOIL (Quinlan 1990) have no predicate invention. In MIL, predicate invention is conducted via construction of substitutions for meta-rules applied by a meta-interpreter. The use of the meta-rules clarifies the declarative bias being employed. New predicate names are introduced as higher-order Skolem constants, a finite number of which are added during every iterative deepening of the search.

\subsection{Logical mechanisms in concept formation and representation}

Mechanisms from logical reasoning are found to play crucial roles in human understanding and conceptualization. Induction has long been shown to be highly related to concept attainment and information processing (Lemke et al. 1967), and abduction also is considered a key mechanism in this context (Hobbs 2004). Recursion plays a similarly prominent role during the process of concept acquisition and meaning making, and has been argued to be a key human ability regarding language and understanding in general (Hauser et al. 2002). Additionally, the capacity to apply recursion is strictly necessary for the representation of infinite concepts (such as, e.g., the concept of an ancestor, or the notion of ordinal numbers). All three mechanisms are also present in MIL. There, induction and abduction, together with predicate invention, are all achieved by way of (higher-order) meta-rules. Owing to the exis- 
tentially quantified variables in the meta-rules, the resulting first-order theories are strictly logical generalisation of the meta-rules.

Learning recursive programs is a technically difficult task in ILP and is not fully supported by general-purpose ILP systems such as Foil (Quinlan and Cameron 1995), Golem (Muggleton and Feng 1992) and Progol (Muggleton 1995). Still, different techniques allow for the induction of recursive programs. For instance CRUSTACEAN (Aha et al. 1994), CLAM (Rios and Matwin 1996), TIM (Idestam-Almquist 1996) and MRI (Furusawa et al. 1997) use inverse entailment based on structural analysis. SMART (Mofizur and Numao 1996) and FILP (Bergadano and Gunetti 1996) use top-down induction of arbitrary Horn clauses, including recursive definitions. However, the search remains incomplete due to restrictions regarding the use of (intensional) background knowledge, as well as pruning techniques. FILP can only induce functional predicates and SMART cannot learn mutually inter-depending clauses. Regarding functional and inductive programming, for example the system Igor1 relies on explanation-based generalization over program traces (Kitzelmann and Schmid 2006). The successor Igor2 (Kitzelmann 2008) can induce recursive functions which depend on additional, invented functions based on the abduction of input-output pairs for some function call [(e.g., in modelling the inductive generalization of rules for domains such as Tower of Hanoi or blocksworld (Schmid and Kitzelmann 2011)]. However, Igor requires the first $k$ examples of a target theory to generalise over a whole class. Esher (Albarghouthi et al. 2013) is a generic and efficient algorithm which interacts with the user via input-output examples, and synthesizes recursive programs implementing intended behaviour. Hence, Esher needs to query an oracle each time a recursive call is encountered to ask for examples.

\section{Framework}

\subsection{General setting}

We assume sets of constants, predicate symbols and first-order variables are denoted $\mathcal{C}, \mathcal{P}, \mathcal{V}$ respectively. We assume definite clause programs to be defined in the usual way. Furthermore we assume a human as possessing background knowledge $B$ expressed as a definite program. We now define the distinction between private and public predicate symbols.

Definition 1 (Public and private predicate symbols) We say a predicate symbol $p \in \mathcal{P}$ found in definite program $P$ is public with respect to a human population $S$ in the case $p$ forms part of the background knowledge of each human $s \in S$. Otherwise $p$ is private.

Now we define Predicate Invention as follows.

Definition 2 (Predicate Invention) In the case background knowledge $B$ of an ILP system is extended to $B \cup H$, where $H$ is a definite program we call predicate symbol $p \in \mathcal{P}$ an Invention iff $p$ is defined in $H$ but not in $B$.

\subsection{Comprehensibility}

Next we provide our operational definition of comprehensibility.

Definition 3 (Comprehensibility, $C(S, P)$ ) The comprehensibility of a definition (or program) $P$ with respect to a human population $S$ is the mean accuracy with which a human $s$ from population $S$ after brief study and without further sight can use $P$ to classify new material sampled randomly from the definition's domain. 
Note this definition allows us to define comprehensibility in a way which allows its experimental determination given a set of human participants. However, in order to clarify the term "after brief study" we next define the notion of inspection time.

Definition 4 (Inspection time $T(S, P)$ ) The inspection time $T$ of a definition (or program) $P$ with respect to a human population $S$ is the mean time a human $s$ from $S$ spends studying $P$ before applying $P$ to new material.

Since, in the previous subsection, we assume humans as having background knowledge which is equivalent to a definite program, we next define the idea of humans mapping privately defined predicate symbols to ones found in their own background knowledge.

Definition 5 (Predicate recognition $R(S, p)$ ) Predicate recognition $R$ is the mean proportion of times a human $s$ from population $S$ gives the correct public name to a predicate symbol $p$ presented as a privately named definition $q$.

For each of these mappings from privately defined predicate symbols to elements from the background knowledge we can now experimentally determine the required naming time.

Definition 6 (Naming time $N(S, p)$ ) For a predicate symbol $p$ presented as a privately named definition $q$ in definite program $P$ the naming time $N$ with respect to a human population $S$ is the mean time a human $s$ from $S$ spends studying $P$ before giving a public name to $p$.

Lastly we provide a simple definition of the textual complexity of a definite program.

Definition 7 (Textual complexity, $S z(P)$ ) The textual complexity $S z$ of a definition of definite program $P$ is the sum of the occurrences of predicate symbols, functions symbols and variables found in $P$.

\subsection{Ultra-Strong Machine Learning}

The following definitions extend those above by describing measures for estimating the degree to which humans can be aided by inspection of the output of a symbolic Machine Learning algorithm. Firstly we define the output of symbolic Machine Learning.

Definition 8 (Machine Learned Program, $M(E)$ ) The program learned from examples $E$ using Machine Learning algorithm $M$ which outputs a symbolic hypothesis in the form of a definition of program.

Unaided human learning from training examples can now be defined as follows.

Definition 9 (Unaided Human Comprehension of Examples, $C(S, E)$ ) The comprehensibility of a definition (or program) $P$ with respect to a human population $S$ is the mean accuracy with which a human $s$ from population $S$ after brief study of an example set $E$ of a hidden target definition can classify new material sampled randomly from the target definition's domain.

Lastly we define machine-aided human learning from training examples.

Definition 10 (Machine-aided Human Comprehension of Examples, $C(S, M(E))$ ) The machine-aided comprehensibility of a definition (or program) $P$ with respect to a human population $S$ is the mean accuracy with which a human $s$ from population $S$ after brief study of a program $M(E)$, learned by a symbolic Machine Learning algorithm $M$ from examples $E$, can classify new material sampled randomly from the target definition's domain. 
Table 1 Mapping defined properties from this section and independent variables in the experiments

\begin{tabular}{lll}
\hline Experiment & Defined property & Experimental variable \\
\hline Expt 1 & Comprehensibility $C, C_{H}, C_{H M}$ & Score \\
& Inspection time $T$ & Time \\
& Recognition $R$ & CorrectNaming \\
& Naming Time $N$ & NamingTime \\
Expt 2 & Unaided comprehension & $\mathrm{O} 1$ \\
& Machine-aided comprehension & $\mathrm{O} 2$ \\
\hline
\end{tabular}

\subsection{Experimental hypotheses}

We are now in a position to define and explain the motivations for the experimental hypotheses to be tested in Sect. 4. Below $C(S, P), T(S, P), R(S, p), N(S, p), S z(P), C(S, E)$, $C(S, M(E))$ are denoted by $C, T, R, N, S z, C_{\mathrm{H}}, C_{\mathrm{HM}}$ respectively. Note $C_{\mathrm{H}}$ and $C_{\mathrm{HM}}$ indicate respectively Comprehension of a Human given data as opposed to Comprehension of a Human given data and a Machine Learning system.

Hypothesis H1, $C \propto \frac{1}{T}$. This hypothesis relates to the idea of using inspection time as a proxy for incomprehension. That is, we might expect humans to take a long time to commit to an answer in the case they find the program hard to understand. As a proxy, inspection time is easier to measure than comprehension.

Hypothesis H2, $C \propto R$. This hypothesis is related to the idea humans understand private predicate symbols, such as $p 1 / 2$, generated during predicate invention, by mapping them to public ones in their own background knowledge.

Hypothesis H3, $C \propto \frac{1}{S z}$. This hypothesis is motivated by the idea that a key property of predicate invention is its ability to compress a description by introducing new predicates which are used multiply within the definition. We are interested in whether the resultant compression of the description leads to increased comprehensibility.

Hypothesis H4, $R \propto \frac{1}{N}$. This hypothesis relates to the idea that if humans take a long time to recognise and publicly name a privately named predicate they are unlikely to correctly identify it. Analogous to H1, this allows naming time to be used as a proxy for recognition of an invented predicate.

Hypothesis H5, $C_{\mathrm{H}} \leq C_{\mathrm{HM}}$. This hypothesis relates to the idea of Ultra-Strong Machine Learning. That is, we might expect humans to perform better on unseen data after having seen a symbolic machine learned definition compared with simply inspecting the training data.

In the next section we describe experiments which test these four hypotheses. Table 1 shows the mapping between the measurable properties defined in this section and the independent variables used in the experiments.

\section{Experiments}

To investigate the hypotheses concerning comprehensibility of concept description in a logical representation we conducted two experiments with human participants. In the first experiment, our main interest was whether making a concept definition more compact by introducing additional predicates in the body of Prolog rules positively impacts compre- 
hensibility. We also explored several aspects related to the use of invented predicates. In the second experiment, we focussed on ultra-strong learning. To examine whether a machine learned hypothesis is operationally effective, we compared performance of participants when they had to classify unseen objects from the domain on their own in contrast to being offered explicit classification rules learned with an ILP approach. In the following, we will present the details of the experiments, introducing the materials, describing the experimental methods, and giving the results.

\subsection{Experiment 1: comprehensibility and predicate invention}

We tested whether classification rules using additional predicates are helpful per se or whether their helpfulness is dependent on the ability of a person to assign a specific meaning to the predicate. Furthermore, we were interested in possible interactions between predicate use and complexity of rules. For this reason, the first experiment involved variations in material and procedure which are introduced in the following.

\subsubsection{Materials}

Material construction is based on the well-known family relations examples used to teach Prolog (Sterling and Shapiro 1994) and also used in the context of ILP(Muggleton et al. 2015). Based on the grandparent/2 predicate, three additional problems were defined: grandfather/2 which is more specific than grandparent/2, greatgrandparent/2 which requires twice as many rules if defined without an additional (invented) predicate, (i.e. has high textual complexity) and the recursive predicate ancestor/ 2 which has low textual but high cognitive complexity (Kahney 1989). Instead of these meaningful names, target predicates are called $p / 2$. The facts provided are identical to the family tree presented in Muggleton et al. (2015). In the rule bodies, either public names (mother, father) (i.e. names which relate to the semantics of family relations) or private names $(q 1 / 2, q 2 / 2)$ were used. Furthermore, programs were either presented with or without the inclusion of an additional (invented) predicate for parent $/ 2$ which was named $p 1 / 2$. The tree and the predicate definitions for the public name space are given in Fig. 3.

In Sect. 3 we defined comprehensibility of a program as the accuracy with which a human can classify new material sampled from the domain. To assess comprehensibility, we defined seven questions for each of the four predicates (see Fig. 4). For five questions, it has to be determined whether a relation for two given objects is true. For two further questions, it has to be determined for which variable bindings the relation can be fulfilled. In addition, an open question was included, where a meaningful name had to be given to predicate $p / 2$ for each of the four problems and, if applicable, also to the additional predicate $p 1 / 2$.

To evaluate the material, we ran a pilot study (March 2016) at Imperial College London with 16 students of computer science with a strong background in programming, Prolog, and logic. The pilot study was conducted as a paper-and-pencil experiment where for each problem first the seven questions had to be answered and afterwards a meaningful name had to be given to the program. In all, 13 out of the 16 students solved each question correctly and most students were able to give the correct public names to all of the programs, regardless of whether they had to work with the public or with the private names. Participants needed about $20 \mathrm{~min}$ for the four problems. Thus, the instructions and the material are understandable and coherent. A very interesting outcome of the study was that about a third of the students made notes on the questionnaires. Some of the notes showed that students first named the target predicates and the invented predicate and then answered the questions. That is, students gave 


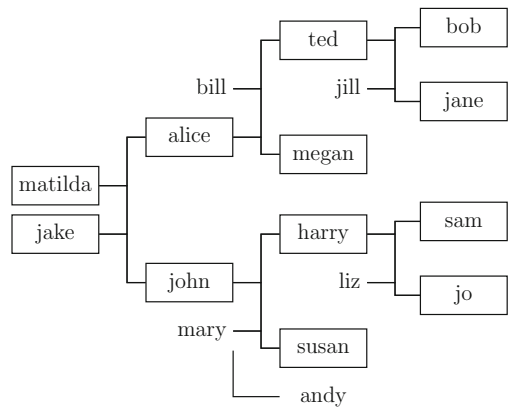

Target Concepts (Rules):

$\%$ grandfather without invented pred.

$\mathrm{p}(\mathrm{X}, \mathrm{Y})$ :- father(X,Z), father(Z,Y).

$\mathrm{p}(\mathrm{X}, \mathrm{Y})$ :- father(X,Z), mother(Z,Y).

$\%$ grandfather with invented predicate $\mathrm{p}(\mathrm{X}, \mathrm{Y})$ :- $\mathrm{p} 1(\mathrm{X}, \mathrm{Z})$, father(Z,Y).

$\mathrm{p} 1(\mathrm{X}, \mathrm{Y})$ :- father(X,Y).

$\mathrm{p} 1(\mathrm{X}, \mathrm{Y})$ :- mother(X,Y).

$\%$ grandparent without invented pred. $\mathrm{p}(\mathrm{X}, \mathrm{Y})$ :- father(X,Z), father(Z,Y).

$\mathrm{p}(\mathrm{X}, \mathrm{Y})$ :- father(X,Z), mother(Z,Y).

$\mathrm{p}(X, Y)$ :- mother(X,Z), mother(Z,Y).

$\mathrm{p}(\mathrm{X}, \mathrm{Y})$ :- mother(X,Z), father(Z,Y).

$\%$ grandparent with invented predicate

$\mathrm{p}(\mathrm{X}, \mathrm{Y})$ :- $\mathrm{p} 1(\mathrm{X}, \mathrm{Z}), \mathrm{p} 1(\mathrm{Z}, \mathrm{Y})$.

$\mathrm{p} 1(\mathrm{X}, \mathrm{Y})$ :- father(X,Y).

p1 $(X, Y):-$ mother $(X, Y)$.

$\%$ ancestor without invented predicate

$\mathrm{p}(\mathrm{X}, \mathrm{Y})$ :- father(X,Y).

$\mathrm{p}(\mathrm{X}, \mathrm{Y})$ :- mother(X,Y).

$\mathrm{p}(\mathrm{X}, \mathrm{Y})$ :- father(X,Z), $\mathrm{p}(\mathrm{Z}, \mathrm{Y})$.

$\mathrm{p}(\mathrm{X}, \mathrm{Y})$ :- mother(X,Z), p(Z,Y).

\section{Background Knowledge (Observations):}

father(jake,alice).

father(jake,john).

father(bill,ted).

father(bill,megan)

father(john,harry).

father(john,susan).

father(ted,bob).

father(ted,jane).

father(harry,sam).

father(harry,jo). mother(matilda,alice). mother(matilda,john). mother(alice,ted). mother(alice,megan). mother(mary,harry). mother(mary,susan). mother(mary,andy). mother(jill,bob). mother(jill,jane). mother(liz,sam). mother(liz,jo).

Fig. 3 Public tree and the Prolog programs for grandfather/2, grandparent/2, greatgrandparent/2, and ancestor/2 with and without use of an additional (invented) predicate parent/2. In the corresponding programs for the private name space, father/2 is replaced by $q 1 / 2$, mother $/ 2$ is replaced by $q 2 / 2$, and given names are replaced by two letter strings as shown in Observations in Fig. 6

Fig. 4 Questions for the grandparent/2 problem with public names
$\%$ greatgrandparent without invented predicate $\mathrm{p}(\mathrm{X}, \mathrm{Y})$ :- father(X,U), father(U,Z), father(Z,Y). $\mathrm{p}(X, Y)$ :- father(X,U), father(U,Z), mother(Z,Y). $\mathrm{p}(\mathrm{X}, \mathrm{Y})$ :- father(X,U), mother(U,Z), father(Z,Y). $\mathrm{p}(\mathrm{X}, \mathrm{Y})$ :- father(X,U), mother(U,Z), mother(Z,Y). $\mathrm{p}(X, Y)$ :- mother(X,U), father(U,Z), mother(Z,Y). $\mathrm{p}(X, Y)$ :- mother(X,U), father(U,Z), father(Z,Y). $\mathrm{p}(\mathrm{X}, \mathrm{Y})$ :- mother(X,U), mother(U,Z), mother $(\mathrm{Z}, \mathrm{Y})$. $\mathrm{p}(\mathrm{X}, \mathrm{Y})$ :- mother(X,U), mother(U,Z), father(Z,Y).

$\%$ greatgrandparent with invented predicate $\mathrm{p}(\mathrm{X}, \mathrm{Y})$ :- $\mathrm{p} 1(\mathrm{X}, \mathrm{U}), \mathrm{p} 1(\mathrm{U}, \mathrm{Z}), \mathrm{p} 1(\mathrm{Z}, \mathrm{Y})$. $\mathrm{p} 1(\mathrm{X}, \mathrm{Y})$ :- father(X,Y). $\mathrm{p} 1(\mathrm{X}, \mathrm{Y})$ :- mother(X,Y).

$\%$ ancestor with invented predicate $\mathrm{p}(\mathrm{X}, \mathrm{Y})$ :- $\mathrm{p} 1(\mathrm{X}, \mathrm{Y})$. $\mathrm{p}(\mathrm{X}, \mathrm{Y})$ :- $\mathrm{p} 1(\mathrm{X}, \mathrm{Z}), \mathrm{p}(\mathrm{Z}, \mathrm{Y})$. $\mathrm{p} 1(\mathrm{X}, \mathrm{Y}):-$ father(X,Y). $\mathrm{p} 1(\mathrm{X}, \mathrm{Y})$ :- mother $(\mathrm{X}, \mathrm{Y})$.
- What is the result of $p$ (bill, bob)? $\square$ true $\quad \square$ false $\quad \square$ don't know

- What is the result of $p$ (jake, harry)? $\square$ true $\quad \square$ false $\quad \square$ don't know

- What is the result of $p$ (bob,bill)? $\square$ true $\quad \square$ false $\quad \square$ don't know

- What is the result of $p(\operatorname{mary}, j o)$ ? $\square$ true $\quad \square$ false $\quad \square$ don't know

- What is the result of $p$ (john, sam)? $\square$ true $\quad \square$ false $\quad \square$ don't know

- What is the result of $p(X, b o b)$ ?
$\square$ false
$\square X=$ bill
$\square X=$ bill; alice
$\square$ don't know
$\square X=$ alice
- What is the result of $p(j \circ h n, X)$ ?
$\square$ false
$\square X=\operatorname{sam}$
$\square X=$ jo
$X=$ sam; jo $\quad \square$ don't know 
a meaningful name without being instructed to do so and one can assume that they used this strategy because it made answering the questions easier.

\subsubsection{Method}

Variables To assess the influence of meaningful names and of predicate invention on comprehensibility, we introduced the following three independent variables:

NameSpace: $\quad$ The name space in which context the problems are presented is either public or private.

PredicateInvention: The problems are given either with or without an additional (invented) predicate $p 1 / 2$ which represents the parent/2 relation.

NamingInstruction: The open question to give a meaningful name to predicate $p / 2$ and - if applicable — also to the additional predicate $p 1 / 2$ is either given before or after the seven questions given in Fig. 4 had to be answered.

The variation of the independent variables results in a $2 \times 2 \times 2$ factor design which was realised between-participants for factors NameSpace and NamingInstruction and within-participants for factor PredicateInvention. Problem presentation with PredicateInvention was either given for the first and the third or the second and the fourth problem.

The textual complexity varies over problems and in dependence of the introduction of the additional predicate $p 1 / 2$. The textually most complex program is greatgrandparent $/ 2$ without the use of $p 1 / 2$. The least complex program is grandfather/2 without the use of $p 1 / 2$ as can be seen in Fig. 3 .

The following dependent variables were assessed:

Score:

Time:

CorrectNaming:

NamingTime:
For each problem, the score is calculated as the sum of correctly answered questions (see Fig. 4). That is, score has minimal value 0 and maximal value 7 for each problem.

The time to inspect a problem is measured from presenting the problem until answering the seven questions.

The correctness of the given public name for a predicate definition $p / 2$ was judged by two measures. Additionally we discriminated between clearly incorrect answers and responses where participants wrote nothing or stated they do not know the correct meaning.

The time for naming is measured from presenting the question until indication that the question is answered by going to the next page. For condition PredicateInvention/with both $p / 2$ and $p l / 2$ had to be named.

Empirical Hypotheses Given the independent and dependent variables, hypotheses can now be formulated with respect to these variables:

H1: Score is inverse proportional to Time, that is, participants who comprehend a program, give more correct answers in less time than such participants who do not comprehend the program.

$\mathrm{H} 2$ : CorrectNaming is proportional to Score, that is, participants who can give the intended public (i.e. meaningful) name to a program have higher scores than participants who do not get the meaning of the program. 
H3: Score is inverse proportional to textual complexity, that is, for problem greatgrandparent/2 the differences of score should be greatest between the PredicateInvention/with and PredicateInvention/without condition because here the difference in textual complexity is highest.

H4: CorrectNaming is inversely proportional to NamingTime, that is, if participants need a long time to come up with a meaningful name for a program, they probably will get it wrong.

Participants and Procedure The experiment was conducted in April 2016 with cognitive science students of the University of Osnabrück. All students had passed at least one previous one-semester course on Prolog programming and all have a background in logic. That is, their background in Prolog is less strong than for the Imperial College sample but they are not novices. From the originally 87 participants, three did not finish the experiment and six students were excluded because they answered "don't know" for more than $50 \%$ of the questions. All analyses were done with the remaining 78 participants (43 male, 35 female; mean age $=23.55$ years, $s d=2.47)^{1}$

The experiment was realised with the soscisurvey.de system and was conducted online during class. After a general introduction, students worked through an example problem ("sibling") to get acquainted with the domain and with the types of questions they needed to answer. Afterwards, the four test problems were presented in one of the experimental conditions. For each problem, on the first page the facts and the tree and the predicate definition was presented. On the next page, this information was given again together with the first question or the naming instruction. If the "next" button was pressed, it was not possible to go back to a previous page.

Working through the problems was self-paced. The four problems were presented in the sequence grandfather/2, grandparent/2, greatgrandparent/2, ancestor/2 for all participants. That is, we cannot control for sequence effects, such as performance gain associated with getting acquainted with the style of the problems and questions or performance loss due to decrease in motivation or fatigue. However, since problem type is not used as an experimental condition, possible sequence effects do not affect statistical analyses of the effects of the independent variables introduced above.

\subsubsection{Results}

Scores and Times When considering time for question answering and naming together, participants needed about $5 \mathrm{~min}$ for the first problem and got faster over the problems. One reason for this speed-up effect might be, that participants needed less time to inspect the tree or the facts for later problems. There is no speed-accuracy trade-off, that is, there is no systematic relation between (low) number of correct answers and (low) solution time for question answering. In the following, time is given in seconds and for statistical analyses time was logarithmically transformed.

Giving meaningful names In the public name condition, the names the participants gave to the programs were typically the standard names, sometimes their inverse, such as "grandchildren", "child of child", or "parent of parent" for the grandparent/2 problem. In the condition with private names, the standard names describing family relations were also used by most participants, however, some participants gave more abstract descriptions, such as "X and $\mathrm{Y}$ are connected via an internode" for grandparent/2. Among

1 A comprehensive description of all analyses and results can be found at http://www.cogsys.wiai.unibamberg.de/publications/comprAnalysesDoc.pdf. 
Table 2 Means and standard deviations of Score in dependence of CorrectNaming, where "no answer" covers answers where participants either did not answer or explicitly stated that they do not know the answer. Results for linear models are given as b-estimates and $p$ values for the contrast between correct and incorrect naming

\begin{tabular}{lllll}
\hline & Correct & Incorrect & No answer & Test \\
\hline Grandfather & $\mathrm{n}=28$ & $\mathrm{n}=46$ & $\mathrm{n}=4$ & \\
Score & Mean $6.68(\mathrm{sd}=0.61)$ & $5.15(1.81)$ & $4.75(1.71)$ & $-1.53, p<0.001$ \\
Grandparent & 50 & 23 & 5 & \\
Score & $6.56(1.23)$ & $5.04(2.12)$ & $3.4(1.82)$ & $-1.52, p<0.001$ \\
Greatgrandparent & 54 & 18 & 6 & $-1, p<0.001$ \\
Score & $6.76(0.66)$ & $5.78(1.66)$ & $3(1.67)$ & \\
Ancestor & 32 & 39 & 7 & $-2.67, p<0.001$ \\
Score & $5.75(1.44)$ & $3.08(1.8)$ & $2.86(1.57)$ & \\
\hline
\end{tabular}

the incorrect answers for the grandparent/2 problem often were over-specific interpretations such as "grandson" or "grandfather". The same was the case for greatgrandparent/2 with incorrect answers such as "greatgrandson". Some participants restricted the description to the given tree, for example, "parent of parent with 2 children" for grandparent/2. Incorrect answers for the ancestor/2 problem typically were overly general, such as "related".

Inverse proportional relation between Score and Time (H1) There is a significant negative Pearsons product-moment correlation between Time and Score over all problems $(r=-.38$, $p \leq 0.001)$.

Effect of CorrectNaming on Score (H2) To assess the impact of being able to give a meaningful name to a problem (CorrectNaming) on comprehensibility (Score), answers were classified as "correct", "incorrect" and "no answer" which covers answers where participants either did not answer or explicitly stated that they do not know the answer. Participants who were able to give meaningful names to the programs answered significantly more questions correctly. Statistical analyses were performed with general linear models with dummy coding (contrast) for the predictor variable CorrectNaming. The results are given in Table 2.

Impact of textual complexity on the effect of PredicateInvention on Score (H3) For the greatgrandparent/2 problem, there is a marginally significant effect of PredicateInvention for NameSpace/private and NamingInstruction/after with a higher score for the PredicateInvention/with condition $(b=-1.59, p=0.09)$.

Relation of CorrectNaming and NamingTime (H4) Participants who give a correct meaningful name to a problem do need less time to do so than participants who end up giving an incorrect name for all problems except ancestor/2. This relation is given in Fig. 5 accumulated over all factors per problem. Statistical analyses were done separately for conditions PredicateInvention/with and PredicateInvention/without because in the first case two names-for target predicate $p / 2$ and for the additional predicate $p 1 / 2$ - had to be given. Differences between correct and incorrect are significant for grandfather/2 in the condition PredicateInvention/without $(b=0.31, p=0.007)$ and marginally significant for grandparent/2 in the condition PredicateInvention/with $(b=0.2, p=0.084)$. For ancestor/ 2 in the condition PredicateInvention/with there is a significant difference between correct naming and "no answer" ( $b=-0.49, p=0.039)$. 


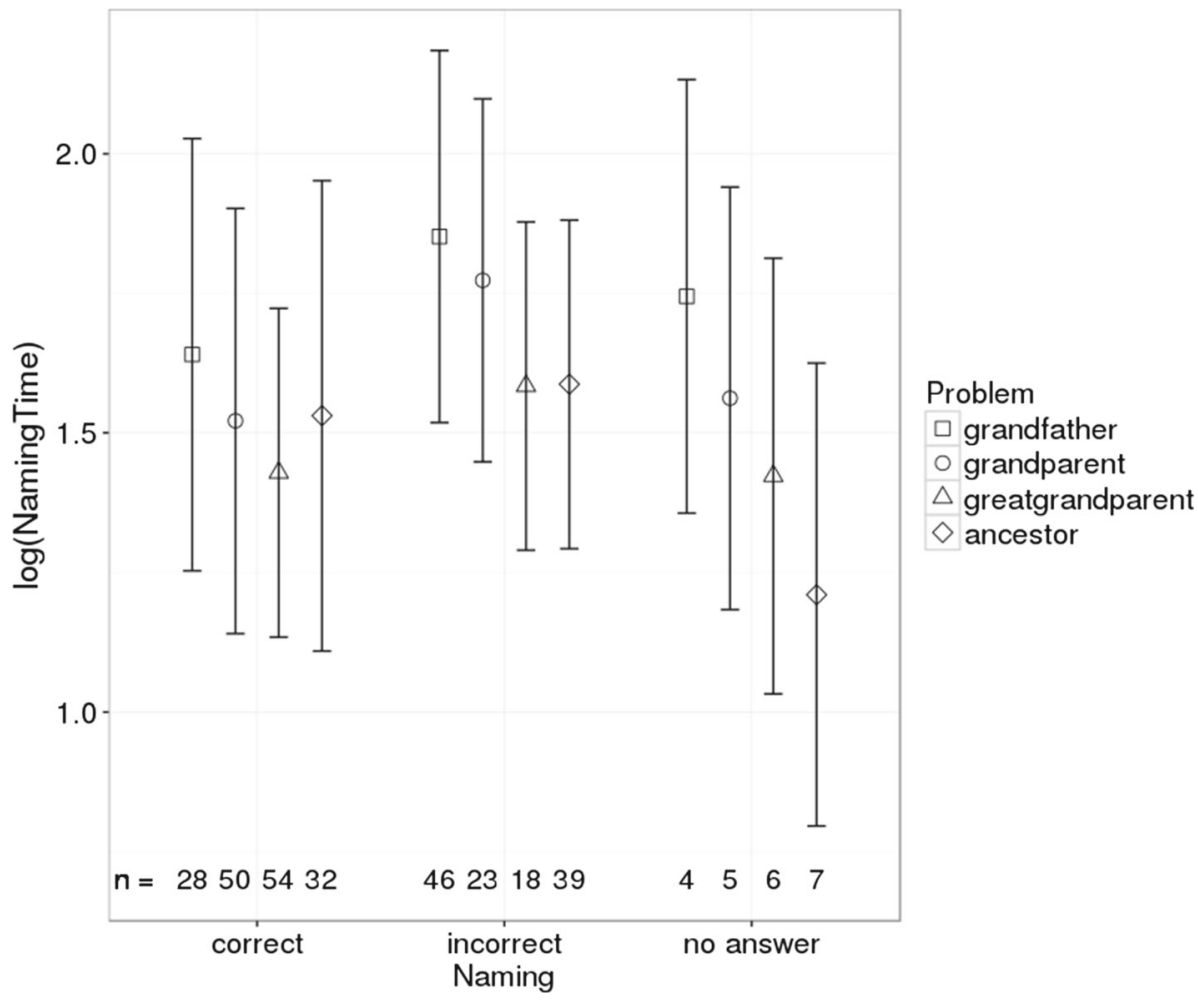

Fig. 5 Relation between time needed for giving a meaningful name and correctness of naming, where "no answer" covers answers where participants either did not answer or explicitly stated that they do not know the answer (averaged over PredicateInvention with/without)

\subsection{Experiment 2: ultra-strong learning}

After exploring the impact of predicate invention on comprehensibility, we conducted a further experiment to test the hypothesis that ILP-learned relational concepts can support humans making sense from observations in complex relational domains. To follow Michie's characterisation of ultra-strong learning, we aim to show that ILP learned classification rules can result in operational effectiveness for humans. That is, given a set of observations in a domain, we need to show that humans are not able to induce a classification rule but an ILP system can and additionally that the ILP learned rules can be understood by humans and successfully applied to new observations. ${ }^{2}$

\subsubsection{Material}

We focused on the grandparent/2 problem investigated in the first experiment (see Fig. 3 ) and constructed an isomorphic fictitious chemistry domain shown in Fig. 6. The Observations correspond to the ones of the private version of the family tree used in Experiment 1 . The Test Results are four positive and negative examples which can be used as training examples for an

${ }^{2}$ A detailed description of the material and the results is given in http://www.cogsys.wiai.uni-bamberg.de/ publications/UltraStrExpAnalyses.pdf. 
Observations:

$$
\begin{array}{ll}
\text { q1(ab,ac). } & \text { q2(aa,ac). } \\
\text { q1(ab,ae). } & \text { q2(aa,ae). } \\
\text { q1(ad,ag). } & \text { q2(ac,ag). } \\
\text { q1(ad,ai). } & \text { q2(ac,ai). } \\
\text { q1(ae,aj). } & \text { q2(af,aj). } \\
\text { q1(ae,al). } & \text { q2(af,al). } \\
\text { q1(ag,an). } & \text { q2(af,am). } \\
\text { q1(ag,ao). } & \text { q2(ah,an). } \\
\text { q1(aj,ap). } & \text { q2(ah,ao). } \\
\text { q1(aj,aq). } & \text { q2(ak,ap). } \\
& \text { q2(ak,aq). }
\end{array}
$$

Today you tested whether a pair of substances are related to an exothermic reaction (a chemical reaction that releases energy by light or heat). For example, exothermic(ac, an) means that ac and an are, respectively, substrate and product of a (chain of) reaction(s) which is exothermic. You observed the following test results:

Test Results:

$$
\begin{array}{ll}
\text { exothermic }(a c, a n) . & \text { not exothermic }(a a, a b) . \\
\text { exothermic }(a a, a l) . & \text { not exothermic }(a d, a i) . \\
\text { exothermic }(a b, a g) . & \text { not exothermic }(a b, a q) . \\
\text { exothermic }(a e, a p) . & \text { not exothermic }(a j, a p) . \\
\text { exothermic }(a a, a g) . & \text { not exothermic }(a n, a c) .
\end{array}
$$

You have a new computer program which can support you in finding rules to characterize substances. When you presented your observations to the program, it returned the following rules:

Rules:

$$
\begin{aligned}
& \text { exothermic }(X, Y):-q 1(X, Z), q 1(Z, Y) . \\
& \text { exothermic }(X, Y):-q 1(X, Z), q 2(Z, Y) . \\
& \text { exothermic }(X, Y):-q 2(X, Z), q 2(Z, Y) . \\
& \text { exothermic }(X, Y):-q 2(X, Z), q 1(Z, Y) .
\end{aligned}
$$

Fig. 6 Fictitious chemistry domain

ILP system such as Metagol. The Rules are the classification rules induced by Metagol. For one group of participants these rules initially are not given and the participants were required to induce them by themselves from the same test results. To assess comprehensibility, an isomorphic questionnaire to the one used in the first experiment has been used (see Fig. 4).

\subsubsection{Method}

Design and Variables To control for possible effects of previous involvement with the problem, we used a pre-test post-test design as shown in Table 3. In a between-participants design, participants of one group were asked to induce a classification rule by themselves from examples (Rule Acquisition and Application, RAA), another got immediately presented with the classification rules analogous to the first experiment (Rule Application, RA). Comprehensibility scores (dependent variable Score) were assessed for both groups after the classification rules were presented $(\mathrm{O} 2)$. For condition RAA, comprehensibility additionally has been assessed after rule induction (O1). For this group, participants additionally were asked to define the classification rule in Prolog or natural language (dependent variable Rule Generation).

Empirical Hypothesis We assume that for this unfamiliar chemistry domain, human problem solvers are not able to come up with the correct classification rules. However, an ILP approach such as Metagol can generate rules which are comprehensible to humans. Consequently, our operational hypothesis is: 
Table 3 Experimental design with conditions Rule Acquisition and Application (RAA) and Rule Application (RA), $\mathrm{R}$ = randomised, $\mathrm{O} 1$ and $\mathrm{O} 2$ are measurements of comprehensibility, $\mathrm{X}$ is the presentation of the ILPlearned rule, $\mathrm{D}$ is an unrelated distractor task

\begin{tabular}{lllll}
\hline RAA & R & O1 & X & O2 \\
RA & $R$ & $D$ & $X$ & O2 \\
\hline
\end{tabular}

H5: Score after simply inspecting training data (O1) is significantly lower than after having seen a symbolic machine learned definition (O2) regardless of whether participants had first to try to induce the rules themselves or not (no difference of $\mathrm{O} 2$ scores between groups RAA and RA).

That is, measurement $\mathrm{O} 1$ addresses unaided human comprehension of examples and $\mathrm{O} 2$ addresses machine-aided human comprehension as introduced in Sect. 3. Additionally, we assume that participants are not able to formulate correct classification rules in Prolog or natural language.

Participants and Procedure The experiment has been conducted in December 2016 at University of Osnabrück. Participants were 43 undergraduate students of cognitive science (20 female, 23 male, mean age $=22.12$ years, $s d=2.51$ ) with a good background in Prolog and in logic but no background in inductive logic programming.

The participants were randomly assigned to one of the following two conditions: Rule Acquisition and Application (RAA, $n=22,12$ male, 10 female, mean age $=22.09$ years, $s d=2.56$ ) or Rule Application (RA, $n=21,11$ male, 10 female, mean age $=22.14$ years, $s d=2.52$ ). For both conditions, participants had to solve comprehensibility problems (O1 only for RAA, O2 for both RAA and RA, see Fig. 4). The participants were tested in class as part of a lecture. The experiment again was realised with soscisurvey.de and participants used their own computers.

After a general introduction, for the RA condition an unrelated task (D in Table 3) was presented to control for length of time and mental effort. Both experimental groups first received an example problem (a blocks-world domain concept) to get acquainted with the experimental task. Then, the participants of the RAA condition were presented with the examples of the chemical domain - but not with the four rules giving the relational concept. Instead, they were asked to describe the concept either in natural language or as Prolog rules. Next, they had to solve the comprehensibility test (O1 in Table 3). From there on, the procedure for the RAA and RA group was identical: Participants were presented with the ILP-learned rules which characterise the searched-for concept and had to solve the second comprehensibility test $(\mathrm{O} 2$ in Table 3 ) which consists of tasks isomorphic to the first test. Afterwards, demographic data were obtained. The experiment took about $20 \mathrm{~min}$.

\subsubsection{Results}

Rule Generation The 22 participants of the RAA condition had to formulate the rules which characterise the target concept exothermic. 13 participants tried to formulate the rules. Of these, 11 wrote Prolog code, 2 gave a natural language description. Only one participant gave the correct rules (in Prolog). All other participants gave erroneous rules, often either too specific (not covering all of the given positive examples) or too general (covering some negative examples). Some example solutions are given in Fig. 7. The results support our assumption that the fictitious chemistry domain is too complex for humans to be able to acquire the correct relational concept from examples. 
Participant 327: too specific

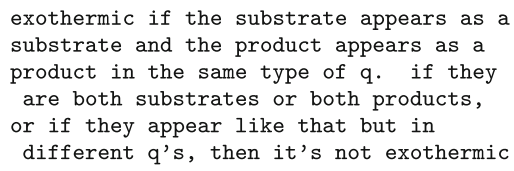

Participant 314: too specific

$\operatorname{exothermic}(X, Y):-q 2(X, Z), q 1(Z, Y)$.

Participant 295: too general

not_exothermic $(X, Y):-q 2(X, Z), q 1(Y, Z)$.

not_exothermic $(X, Y):-q 1(X, Y)$.

$\operatorname{exothermic}(X, Y):-\operatorname{not}\left(\operatorname{not} \_\operatorname{exothermic}(X, Y)\right)$.

Fig. 7 Examples for erroneous rules

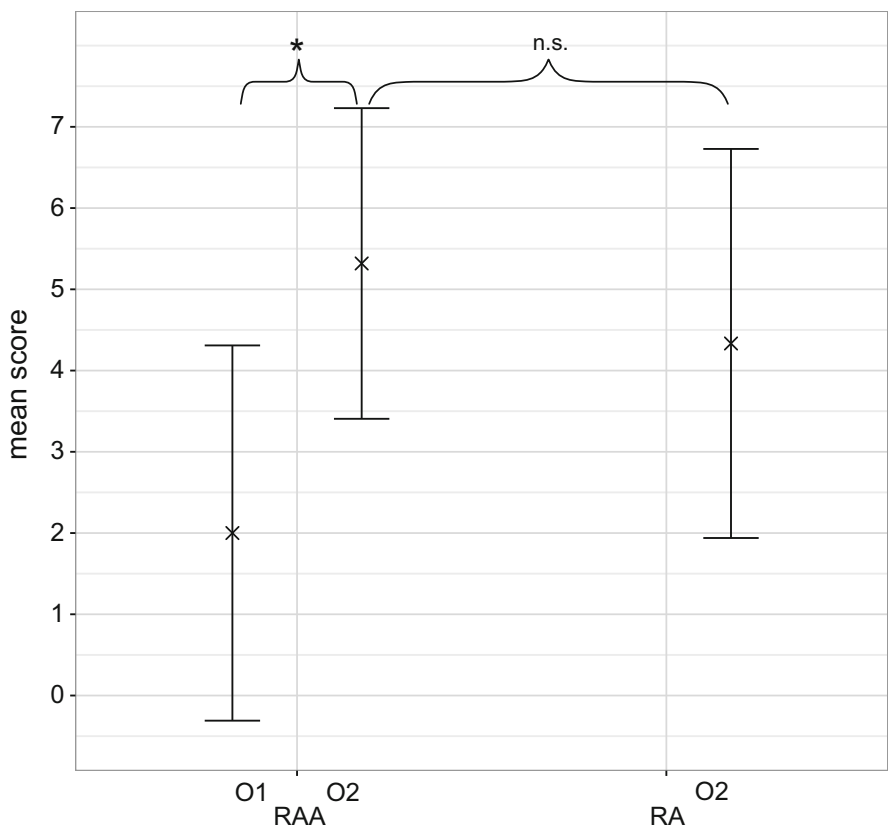

Fig. 8 Mean comprehensibility scores for rule acquisition and application (RAA) versus rule application (RA) condition (details see text)

Scores To evaluate the comprehensibility scores, we excluded the one participant who could formulate the correct relational concept. This participant also had maximum score values for both comprehensibility tests. Participants of the RAA condition had very low comprehensibility scores at the first testing time $(n=21$, mean $=1.76, s d=2.07)$. However, their scores significantly improved for the second testing time (t-test for dependent samples, $t(21)=7.63, p<0.001)$, that is, after they were presented with the ILP-learned rules $(n=21$, mean $=5.24, s d=1.92)$. Participants of the RA condition who immediately were presented the ILP-learned rules performed slightly worse $(n=21$, mean $=4.33$, $s d=2.39$ ), but not significantly so (Wilcoxon rank sum test with continuity correction, $W=267, p=0.119$ ). The results are summarised in Fig. 8. They clearly support our hypothesis that white box Machine Learning approaches such as ILP can support humans to identify relational concepts in complex domains.

\subsection{Discussion}

In Experiment 1 our results show that presenting programs in relation to a public name space facilitates comprehension. Contrary to expectations, being instructed to first think about a 
Table 4 Hypotheses concerning comprehensibility, meaningful names, and predicate invention. Conf. stands for Confrimation, $\mathrm{C}$ means confirmed, $\mathrm{P}$ partially confirmed

\begin{tabular}{lll}
\hline Hypothesis & Conf. \\
\hline H1 & Comprehensibility manifests itself in high scores and fast solution times. & C \\
H2 & Comprehensibility means to be able to give a meaningful name to a program. & C \\
H3 & Predicate invention helps comprehensibility if it reduces textual complexity of the program. & P \\
H4 & If coming up with a meaningful name needs a long time, it will probably be the false concept. & P \\
H5 & ILP can generate classification rules which fulfil the ultra-strong learning criterion. & C \\
\hline
\end{tabular}

meaningful name for a program before answering questions does not facilitate generation of answers. We expected that having a (denotational) semantic interpretation for a predicate supports working on classification and variable bindings of new material from a given domain because mental evaluation of a program can be — at least partially—avoided. Furthermore, the use of additional (invented) predicates does not facilitate program comprehension in general but only under specific conditions which are discussed below (H3).

Results concerning our hypotheses are summarised in Table 4. Hypothesis H1 is confirmed by our empirical data: if a person comprehends a program, she or he can come up with correct answers in short time. Hypothesis $\mathrm{H} 2$ is also confirmed: participants who can give a meaningful name to a program give more correct answers than participants who give incorrect answers or state that they do not know the answer. In addition, participants who give a correct name give answers faster. However, this result can also interpreted as being better able to solve the classification tasks in the comprehensibility test influences better predicate recognition. As hypothesis $\mathrm{H} 3$ we assumed that predicate invention supports comprehensibility if it reduces the textual complexity of a program. For the four problems we investigated, the reduction in complexity is greatest for greatgrandparent/2. Here we get a partial confirmation: predicate invention results in more correct answers for the private name space and if the instruction for naming was given after question answering. This experimental condition is the most challenging, because comprehensibility is not supported by public names and because participants were not encouraged to think about the meaning of the presented predicate before they had to answer questions.

Finally, we assumed that persons who have problems in coming up with a meaningful name for a predicate spend a longer amount of time to come up with an (incorrect or no) answer (H4). Results show that this is the case-with the exception of the ancestor/2 problem. However, the differences are only significant under specific conditions. The observation that long answering time can indicate a problem with comprehensibility could be exploited for the design of the interaction of a person with an ILP system: if a person does not come up quickly with a name for a predicate's the system could offer examples of the predicates behaviour. For example, for the ancestor/2 problem, pairs for which this predicate is true could be highlighted.

While in Experiment 1 the contribution of predicate invention to comprehensibility was explored with respect to the above discussed contexts, in Experiment 2 we focussed on the core characterisics of ultra-strong learning, that is, on operational effectiveness. Indeed, the results of Experiment 2 show that in cases where humans are not able to induce the underlying relational concept of complex domain, they nevertheless can correctly apply an explicit, rule-based representation of the relational concept (H5). All together, our empirical 
results indicate that inductive logic programming can be used to help humans to make sense of complex data.

\section{Conclusions and further work}

In this paper we provide an operational definition of comprehensibility of a logic program (Sect. 3) and use this within within two experiments.

In the first experiment we identify factors which affect comprehension. These factors include the time required to inspect the program, the accuracy with which a partcipant can recognise a predicate to one already known and the textual complexity of the program. As expected, the four problems differ with respect to comprehensibility. The problem most participants had difficulty with is the recursive ancestor/2. For this problem less than half of the participants (32) gave the correct meaningful name and for this problem participants have the lowest scores.

The second experiment tested whether humans can improve performance on unseen data when shown a program generated by a symbolic machine learning system compared with their predictions based only on studying the training data. The experimental support of hypothesis $H 5$ represents a world first demonstration of the existence of Ultra-Strong Machine Learning in Michie's (1988) sense. However, for further work we note H5 will only hold when Machine Learning is effective.

While it is encouraging that our hypotheses do not need to be rejected given the results of the two experiments, further empirical studies are necessary to strengthen our results. Currently, we are conducting an experiment with participants without background in logic and programming, with a "translation" of the Prolog problems to natural language.

In further work we hope to better characterise properties of learned programs which improve ease of comprehension. The aim is to provide guidelines for Machine Learning developers, particular within ILP, to allow human knowledge and skills to be advanced by systems whose comprehensibility properties are optimised. Better understanding of these properties should inform better human-interface design for Machine Learning systems. However, we also hope in the long term to provide clear criteria for optimising search functions in Machine Learning systems to identify and suggest hypotheses which are easy for humans to understand. We believe such systems will be of value not only in scientific discovery assistants, where readable hypotheses are critical, but also in machine learned skill-improvement assistants for improving human performance in tasks such as sports, musical performance or recovery from injury and illness.

In closing we believe the operational definition of comprehensibility has enormous potential to both clarify one of the central concepts of AI research as well as to provide a bridge to the study of factors affecting the design of AI systems which improve human understanding.

Open Access This article is distributed under the terms of the Creative Commons Attribution 4.0 International License (http://creativecommons.org/licenses/by/4.0/), which permits unrestricted use, distribution, and reproduction in any medium, provided you give appropriate credit to the original author(s) and the source, provide a link to the Creative Commons license, and indicate if changes were made. 


\section{References}

Aha, D. W., Lapointe, S., Ling, C. X., \& Matwin, S. (1994). Inverting implication with small training sets. In European conference on machine learning (pp. 29-48). Springer.

Albarghouthi, A., Gulwani, S., \& Kincaid, Z. (2013). Recursive program synthesis. In International conference on computer aided verification (pp. 934-950). Springer.

Allahyari, H., \& Lavesson, N. (2011). User-oriented assessment of classification model understandability. In 11th Scandinavian conference on artificial intelligence. IOS Press.

Askira-Gelman, I. (1998). Knowledge discovery: Comprehensibility of the results. In Proceedings of the thirty-first Hawaii international conference on system sciences (Vol. 5, pp. 247-255). IEEE.

Bergadano, F., \& Gunetti, D. (1996). Inductive logic programming: From machine learning to software engineering. Cambridge: MIT Press.

Chandrasekaran, B., Tanner, M. C., \& Josephson, J. R. (1989). Explaining control strategies in problem solving. IEEE Expert, 4(1), 9-15.

Clancey, W. J. (1983). The epistemology of a rule-based expert system: A framework for explanation. Artificial Intelligence, 20(3), 215-251.

Doran, D., Schulz, S., \& Besold, T. R. (2017). What does explainable ai really mean? A new conceptualization of perspectives. arXiv preprint arXiv: 1710.00794.

Forbus, K. D. (2016). Software social organisms: Implications for measuring AI progress. AI Magazine, 37(1), 85-90.

Freitas, A. A. (2014). Comprehensible classification models: A position paper. SIGKDD Explorations Newsletter, 15(1), 1-10.

Furusawa, M., Inuzuka, N., Seki, H., \& Itoh, H. (1997). Induction of logic programs with more than one recursive clause by analyzing saturations. In International conference on inductive logic programming (pp. 165-172). Springer.

Gaines, B. R. (1996). Transforming rules and trees into comprehensible knowledge structures. In U. M. Fayyad, G. Piatetsky-Shapiro, P. Smyth \& R. Uthurusamy (Eds.), Advances in knowledge discovery and data mining (pp. 205-226). Palo Alto, CA: AAAI Press.

Hauser, M. D., Chomsky, N., \& Fitch, W. T. (2002). The faculty of language: What is it, who has it, and how did it evolve? Science, 298(5598), 1569-1579.

Hobbs, J. R. (2004). Abduction in natural language understanding. In L. R. Horn \& G. Ward (Eds.), Handbook of pragmatics (pp. 724-741). Blackwell.

Huysmans, J., Dejaeger, K., Mues, C., Vanthienen, J., \& Baesens, B. (2011). An empirical evaluation of the comprehensibility of decision table, tree and rule based predictive models. Decision Support Systems, 51(1), 141-154.

Idestam-Almquist, P. (1996). Efficient induction of recursive definitions by structural analysis of saturations. In L. De Raedt (Ed.), Advances in inductive logic programming (pp. 192-205). Amsterdam: IOS Press.

Kahney, H. (1989). What do novice programmers know about recursion? In E. Soloway \& J. C. Spohrer (Eds.), Studying the novice programmer (pp. 209-228). Mahwah: Lawrence Erlbaum.

Kedar-Cabelli, S. T., \& McCarty, L. T. (1987). Explanation-based generalization as resolution theorem proving. In P. Langley (Ed) Proceedings of the fourth international workshop on machine learning, Los Altos (pp. 383-389). Morgan Kaufmann.

Kitzelmann, E. (2008). Analytical inductive functional programming. In International symposium on logicbased program synthesis and transformation (pp. 87-102). Springer.

Kitzelmann, E., \& Schmid, U. (2006). Inductive synthesis of functional programs: An explanation based generalization approach. Journal of Machine Learning Research, 7, 429-454.

Lakkaraju, H., Bach, S. H., \& Leskovec, J. (2016). Interpretable decision sets: A joint framework for description and prediction. In Proceedings of the 22nd ACM SIGKDD international conference on knowledge discovery and data mining (pp. 1675-1684). ACM.

Lemke, E. A., Klausmeier, H. J., \& Harris, C. W. (1967). Relationship of selected cognitive abilities to concept attainment and information processing. Journal of Educational Psychology, 58(1), 27.

Letham, B., Rudin, C., McCormick, T. H., \& Madigan, D. (2015). Interpretable classifiers using rules and bayesian analysis: Building a better stroke prediction model. Annals of Applied Statistics, 9(3), 13501371.

Lim, B. Y., Dey, A. K., \& Avrahami, D. (2009). Why and why not explanations improve the intelligibility of context-aware intelligent systems. In Proceedings of the SIGCHI conference on human factors in computing systems, CHI '09, New York, NY, USA (pp. 2119-2128). ACM.

Lipton, Z. C. (2016). The mythos of model interpretability. CoRR, abs/1606.03490. 
Lou, Y., Caruana, R., \& Gehrke, J. (2012). Intelligible models for classification and regression. In Proceedings of the 18th ACM SIGKDD international conference on knowledge discovery and data mining (pp. 150158). ACM.

Michie, D. (1988). Machine learning in the next five years. In Proceedings of the third European working session on learning (pp. 107-122). Pitman.

Mitchell, T. M., Keller, R. M., \& Kedar-Cabelli, S. T. (1986). Explanation-based generalization: A unifying view. Machine Learning, 1(1), 47-80.

Mofizur, C. R., \& Numao, M. (1996). Top-down induction of recursive programs from small number of sparse examples. In L. De Raedt (Ed.), Advances in inductive logic Programming (pp. 236-253). Amsterdam: IOS Press.

Mozina, M., Zabkar, J., \& Bratko, I. (2007). Argument based machine learning. Artificial Intelligence, 171(1015), 922-937.

Muggleton, S. H. (1995). Inverse entailment and Progol. New Generation Computing, 13, 245-286.

Muggleton, S. H., \& Buntine, W. (1988). Machine invention of first-order predicates by inverting resolution. In Proceedings of the 5th international conference on machine learning (pp. 339-352). Kaufmann.

Muggleton, S. H., De Raedt, L., Poole, D., Bratko, I., Flach, P., \& Inoue, K. (2011). ILP turns 20: Biography and future challenges. Machine Learning, 86(1), 3-23.

Muggleton, S. H., \& Feng, C. (1992). Efficient induction of logic programs. In S. H. Muggleton (Ed.), Inductive logic programming (pp. 281-298). London: Academic Press.

Muggleton, S. H., Lin, D., Pahlavi, N., \& Tamaddoni-Nezhad, A. (2014). Meta-interpretive learning: Application to grammatical inference. Machine Learning, 94, 25-49.

Muggleton, S. H., Lin, D., \& Tamaddoni-Nezhad, A. (2015). Meta-interpretive learning of higher-order dyadic datalog: Predicate invention revisited. Machine Learning, 100(1), 49-73.

Murphy, G. L., \& Lassaline, M. E. (1997). Hierarchical structure in concepts and the basic level of categorization. In K. Lamberts \& D. Shanks (Eds.), Knowledge, concepts, and categories (pp. 93-131). Cambridge: Psychology Press, MIT Press.

Quinlan, J. R. (1990). Learning logical definitions from relations. Machine Learning, 5, 239-266.

Quinlan, J. R., \& Cameron, R. M. (1995). Induction of logic programs: FOIL and related systems. New Generation Computing, 13, 287-312.

Rios, R., \& Matwin, S. (1996). Efficient induction of recursive prolog definitions. In Conference of the Canadian Society for Computational Studies of Intelligence (pp. 240-248). Springer.

Rouveirol, C., \& Puget, J.-F. (1989). A simple and general solution for inverting resolution. In EWSL-89 (pp. 201-210). London: Pitman.

Rudin, C. (2014). Algorithms for interpretable machine learning. In Proceedings of the 20th ACM SIGKDD international conference on knowledge discovery and data mining, KDD '14, New York, NY, USA (pp. 1519-1519). ACM.

Schielzeth, H. (2010). Simple means to improve the interpretability of regression coefficients. Methods in Ecology and Evolution, 1(2), 103-113.

Schmid, U., \& Kitzelmann, E. (2011). Inductive rule learning on the knowledge level. Cognitive Systems Research, 12(3), 237-248.

Schmid, U., Zeller, C., Besold, T., Tamaddoni-Nezhad, A., \& Muggleton, S. H. (2017). How does predicate invention affect human comprehensibility? In A. Russo \& J. Cussens (Eds.) Proceedings of the 26th international conference on inductive logic programming (ILP 2016, September 4th-6th, London). Springer

Shortliffe, E. H. (1974). A rule-based computer program for advising physicians regarding antimicrobial therapy selection. In Proceedings of the 1974 annual ACM conference-volume 2 (pp. 739-739). ACM.

Srinivasan, A. (2001). The ALEPH manual. Oxford: Machine Learning at the Computing Laboratory, Oxford University.

Stahl, I. (1992). Constructive induction in inductive logic programming: An overview. Technical report, Fakultat Informatik, Universitat Stuttgart.

Sterling, L., \& Shapiro, E. Y. (1994). The art of Prolog: Advanced programming techniques. Cambridge: MIT Press.

Turing, A. M. (1950). Computing machinery and intelligence. Mind, 59(236), 433-460.

Van Someren, M. (1995). A perspective on machine learning and comprehensibility from knowledge acquisition. In Proceedings of the workshop on comprehensibility in machine learning, IJCAI-95. Citeseer.

Vellido, A., Martín-Guerrero, J. D., \& Lisboa, P. J. G. (2012). Making machine learning models interpretable. In Proceedings of the 20th european symposium on artificial neural networks, computational intelligence and machine learning ESANN 2012 (pp. 163-172).

Wick, M. R., \& Thompson, W. B. (1992). Reconstructive expert system explanation. Artificial Intelligence, 54(1-2), 33-70. 